\title{
Proceedings
}

Juntendo Medical Journal

2016. 62 (Suppl 1), 6-11

\section{The Cutting Edge in Brain Science and Sportology}

\author{
YASUSHI MIYASHITA \\ *Juntendo University Graduate School of Medicine, Tokyo, Japan
}

\begin{abstract}
Objective: We aimed to establish a novel approach that enables the prediction of behavioral effects of lesions using neuroimaging data, which is applicable to sportological analyses, particularly in identifying the links between brain activity and behavior related to sports.

Materials and Methods: Experimental details are described in our previous reports (Miyamoto et al., 2013; Osada et al., 2015). Four monkeys (M. mulatta and M. fuscata) were used. All experimental protocols, animal welfare, and steps for minimizing suffering were in full compliance with the Guidelines for Proper Conduct of Animal Experiments by Science Council of Japan.

Functional images were acquired using a 4.7-T MRI scanner (Biospec47/40, Bruker) during a recognition or temporal-order judgment task. Monkeys performed the tasks while sitting in an MRI-compatible chair. Group analyses of image data were conducted using SPM5.

Results: A task-evoked connectivity network was identified based on data from the functional imaging conducted during recognition tasks (Miyamoto et al., 2013) and temporal-order judgment tasks (Osada et al., 2015). A significant positive correlation was observed between the predicted impact on performance and the betweenness centrality based on task-evoked connectivity network, whereas no correlation was observed between the predicted impact on performance and the betweenness centrality based on anatomical connectivity network.

Conclusions: Severity of impairment arising from local brain damage was predicted by network "hubness" of the damaged area in the task-evoked connectivity network. These findings will form the basis for future research that aims to predict the functional brain center for watching or partaking in sports.
\end{abstract}

Key words: sportology, brain science, neuroimaging, network hub, animal model

\section{Introduction}

Sportology is a new integrated scientific approach that aims to clarify the relationship between sports and health ${ }^{1)}$. To this end, sportology combines basic and clinical medical sciences, including brain science/neuroscience, and uses cutting-edge methodologies to aid the development of a new scientific approach. In this article, I will review some of our recent attempts to achieve this goal. I will also outline the potential for brain science/neuroscience to contribute to sportology through the development of a new methodology that allows brain imaging data to predict behavioral outcomes.
Sport has an impressive ability to encourage and motivate people, even in their darkest hours. For example, after the catastrophic earthquake and tsunami which devastated Japan on March 11, 2011, the victory of 'Nadeshiko Japan' (Japan women's national football team) in 2011 FIFA Women's World Cup strongly encouraged us, even though most of us only watched it on television. How does sport move us so strongly?

The neuronal and physiological mechanisms by which we are encouraged, moved, and comforted have received much attention in neuroscience. In particular, neuroimaging has identified several brain regions that may contribute to such neuronal

\footnotetext{
Yasushi Miyashita

Juntendo University Graduate School of Medicine

2-1-1 Hongo, Bunkyo-ku, Tokyo 113-8421, Japan

TEL: +81-3-5802-1547 E-mail: y-miyashita@juntendo.ac.jp

The $2^{\text {nd }}$ Congress, International Academy of Sportology 〔Held on Sep. 12, 2015〕

〔Received Dec. 1, 2015]
} 
processes ${ }^{2)}{ }^{3)}$, including, for example, the amygdala, nucleus accumbens, cingulate cortex, orbitofrontal cortex, hypothalamus, and widely distributed cortical areas. However, not all of these areas causally control the neural processes that eventually have impact on behavior (including emotion). Indeed, the severity of the emotional deficit after a lesion in these areas largely depends on which of these identified areas is damaged; lesions in only a fraction of the activated areas actually lead to emotional deficits. Why some areas activated in neuroimaging are "lesion effective" and others are not is currently unknown.

Recently, we developed a novel approach to identify task-related brain networks ${ }^{4)}$ and this approach was extended so that it allows behavioral consequences of brain damage to be precisely predicted in primates ${ }^{5)}$. Functional magnetic resonance imaging (fMRI) was used to measure whole-brain activity during the performance of a cognitive task (in our proof-of-concept experiments, we used well-established memory tasks). Task-specific activation was seen in specific areas, and functional connectivity between them formed a hierarchical network centered on a hub. The task-specific "functional hub" in this dynamic network was found to accurately correspond to the well-documented lesion-effective site ${ }^{6}$, and to exclude the neighboring non-lesion-effective sites. Quantitatively, the predicted severity of behavioral impairment was proportional to the network "hubness" of the lesioned area in the functional network, rather than in the anatomical network. The "hubness" was measured by the index of "betweenness centrality," as we will discuss later in this article (Note that the betweenness centrality of a network node is defined as the fraction of shortest paths passing a given node in the network ${ }^{7)}$ ). These results suggest that the brain areas dynamically shape a hub-centric network and reallocate the lesion-effective site apart from the static anatomical hubs, depending on the specific cognitive demands. This novel approach could thus allow neuroimaging data to be used for the prediction of behavioral outcomes of lesions. This has implications for sportological analyses of behaviors that arise when we play or watch sports.

\section{Materials and methods}

All the experimental protocols, animal welfare, and steps for ameliorating sufferings were in full compliance with the Guidelines for Proper Conduct of Animal Experiments by the Science Council of Japan and with the NIH Guidelines for the Care and Use of Laboratory Animals.

In order to develop this new methodology, we used a well-documented memory system in monkeys ${ }^{2)}{ }^{3)}$. Experimental details are described in our previous paper by Miyamoto et al. (2013) and Osada et al. (2015). In this experiment, two monkeys performed a recognition task $^{4)}$ 8) or a temporal-order judgment task $^{6)}$ modified for $\mathrm{fMRI}^{5}$. . In a custom-made MRI-compatible monkey chair (Nakazawa, Tokyo, Japan), each monkey manipulated an optical fiber-based, custom-made three-way joystick with one of its forelimbs. When the monkey became ready, a list of visual stimuli was presented serially (Cue). The stimuli were selected in a pseudorandom order from a pool of 1,200 pictures of natural or artificial objects. The last stimulus was followed by a delay period (Delay). Finally, two stimuli were simultaneously presented (Choice). In the temporal-order judgment task, the choice stimuli were from the studied list and, if the monkey responded by moving the joystick in the direction of the stimulus that had been presented more recently, the monkey received juice drops. In the recognition task, one choice stimuli was from the studied list and the other was a new stimulus and, if the monkey responded by moving the joystick in the direction of the new stimulus, the monkey received juice drops. Incorrect choices resulted in termination of the trial without reward. Trials were separated by an inter-trial interval, during which the screen was black. In the judgment stage of the temporal-order judgment task, two types of trials were performed: (1) trials in which the choice stimulus pair included neither the initial nor last end stimuli in the list (MIDDLE trial) and (2) trials in which the paired stimuli were both the initial and last end stimuli in the list (BOTH-END trial).

Functional images were acquired and processed as described previously ${ }^{2)}{ }^{4)}$ ). In brief, the monkeys were scanned in a 4.7-T MRI scanner (Biospec 47/40, Bruker, Ettlingen, Germany) with $100 \mathrm{mT} / \mathrm{m}$ 
actively shielded gradient coils and a transceiver saddle RF coil (Takashima, Tokyo, Japan). In each session, functional data were acquired using a gradient-echo echo-planar imaging (EPI) sequence $\left(\mathrm{TR}=2.5 \mathrm{~s}, \mathrm{TE}=20 \mathrm{~ms}, 1.25 \times 1.5 \mathrm{~mm}^{2}\right.$ in-plane resolution, $64 \times 96$ matrix, slice thickness $=1.5 \mathrm{~mm}$ ). T2-weighted spin-echo (RARE) images with the same geometry as the EPI were also scanned. In separate sessions, high-resolution T1-weighted structural images were scanned using a $3-D$ MDEFT sequence $(0.5 \mathrm{~mm}$ isotropic). High-resolution EPI $(32-$ shot, TR $=2.5 \mathrm{~s}, \mathrm{TE}=20 \mathrm{~ms}, 0.625 \times$ $0.75 \mathrm{~mm}^{2}$ in-plane resolution, $128 \times 192$ matrix, slice thickness $=0.8 \mathrm{~mm}$ ) was also acquired to serve as the template image for spatial normalization.

The group analysis of the image data was conducted using a random effect model by treating each session's data from both monkeys equally as a random effect. The regions active during temporalorder judgment were identified by comparison of blood-oxygenation-level-dependent (BOLD) signals between the MIDDLE and BOTH-END conditions. Psychophysiological interaction (PPI) was estimated among the activated areas to determine whether the temporal-order retrieval load affects the connectivity among the identified areas. The effect size of the PPI at target ROIs was evaluated as the beta estimate for the PPI predictor averaged across all sessions for the two monkeys.

The topology of the connectivity networks among the activated areas were examined by calculating network metrics ${ }^{57}$. For each area (node), the network measure of "betweenness centrality" was computed. The betweenness centrality is the fraction of shortest paths passing a given node. In the analysis, the distance from node $A$ to node $B\left(d_{A^{->B}}\right)$ was calculated as the inverse of the PPI $z^{-}$value from node $A$ to node $B\left(d_{A->B}=\right.$ $1 / \mathrm{PPI}_{A->B}$ ).

We used Multi-Voxel Pattern Analysis (MVPA) based on Support Vector Machine (SVM) ${ }^{5)}$ 9) to determine whether the PPI connectivity pattern encodes behavioral performance. Details are described in Osada et al. (2015). In brief, the PPI beta-values (MIDDLE > BOTH-END) for each connection in each session from the high and low performance dataset were analyzed using SVM. SVM discriminated between sessions that belonged to two different classes (high or low performance set), using nonlinear radial basis functions $(f(\mathbf{x}, \mathbf{y})=$ $\exp \left(-\gamma|\mathbf{x}-\mathbf{y}|^{2}\right), \mathbf{x}, \mathbf{y}$ : training vectors $)$.

To computationally examine whether removal of an area affected prediction accuracy, we conducted an SVM prediction analysis using the node-deleted PPI connectivity patterns. The prediction accuracy of the node-deleted PPI connectivity patterns for each node was estimated and compared with that using all the connectivity patterns. A reduction in prediction accuracy was defined as "predicted impact on performance". The predicted impact on performance was calculated for each node for each monkey. To examine the relationship between betweenness centrality and predicted impact on performance, we performed an ANCOVA on predicted impact on performance after removal of each area (monkey $\times$ betweenness centrality). Betweenness centrality and predicted impact on performance after removal of each area were calculated for each monkey and were used for the analysis.

Results

The activation analysis and PPI analysis revealed a brain network that is engaged during performance of the tasks. Figure- 1 shows the task-evoked connectivity network for the temporal-order judgment task. In this figure, only PPIs with $\mathrm{p}<0.01$ (FDR correction) are displayed as directed edges. The diameter of each node represents the betweenness centrality value. These results demonstrate that area $9 / 46 \mathrm{~d}$ acted as a hub in the task-evoked connectivity network during the temporal-order judgment task. When we evaluated the betweenness centrality of the areas based on the directed axonal projection pattern (i.e., anatomical network) with the aid of the CoCoMac database ${ }^{4)}$ ), the betweenness centralities of areas 8Ad and LIP were statistically significant, whereas that of area $9 / 46 \mathrm{~d}$ was not. Thus, structurally, the cortical areas contributing to this task form a network centered at areas 8Ad and LIP as anatomical hubs. The difference in location between functional and anatomical hubs suggests that there is a dynamic allocation of the functional hub that is dependent on the cognitive demands. Interestingly, the task-specific functional hub in this dynamic task-evoked network accurately corresponded to the well-documented 


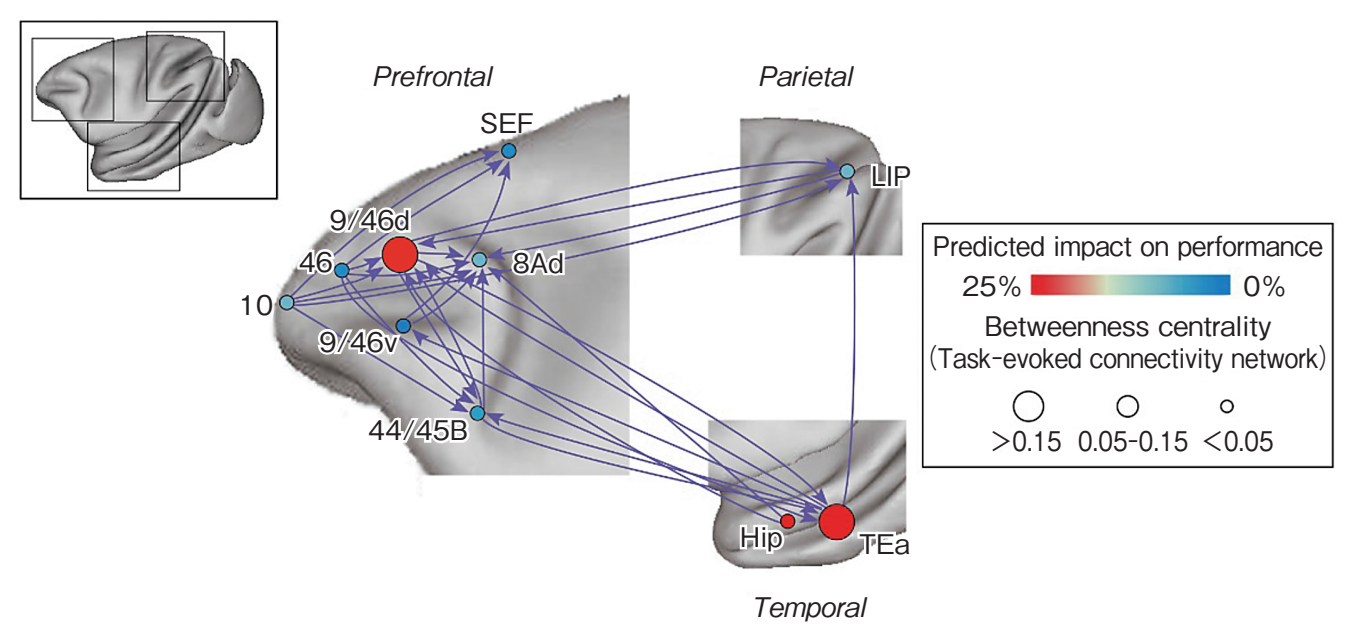

Figure-1 Activated areas and interareal connections in the temporal-order judgment task Psychophysiological Interactions (PPI) with $\mathrm{p}<0.01$ (FDR correction) are displayed as directed edges for display purpose. Node color indicates predicted impact on performance. Node diameter represents betweenness centrality. (Modified from Figure-6D of Osada et al., PLoS Biol, 2015; 13: e1002177 ${ }^{5)}$ )

lesion-effective site, and did not include the neighboring non-lesion-effective site (i.e., severe temporal-order memory impairment after lesions in area $9 / 46$ d, but not in area 8 Ad) ${ }^{5)}$ ).

Figure-1 also shows that a large value of "predicted impact on performance" (\%, color coded) was given to area $9 / 46 \mathrm{~d}(\mathrm{p}=0.03$, randomization test), indicating a significant performance drop after removal of the $9 / 46 \mathrm{~d}$ node. On the other hand, removal of area $8 \mathrm{Ad}$ did not cause a significant reduction in prediction accuracy $(\mathrm{p}=0.59$, randomization test). These results indicate that the areal dissociation of lesion-induced impairment was predicted by the node-deleted PPI connectivity pattern.

We thus compared the predicted impact on performance after lesioning and the betweenness centrality based on the task-evoked connectivity network for each area in each individual monkey. An analysis of covariance (ANCOVA) on predicted impact on performance after removal of each area (monkey $\times$ betweenness centrality) revealed a significant main effect of betweenness centrality of the removed area $(F(1,16)=6.65, p=0.02)$. There was no significant main effect of monkey $(\mathrm{F}(1,16)=$ $0.22, \mathrm{p}=0.64)$, and no interaction between monkey and betweenness centrality $(\mathrm{F}(1,16)=0.85, \mathrm{p}=$ $0.36)$. Importantly, we found a significant positive correlation between the betweenness centrality and the predicted impact on performance $(r=0.53, p=$
0.008) (Figure-2A). These observations indicate that removal of an area with higher betweenness centrality in the task-evoked connectivity network causes a larger reduction in prediction accuracy. On the contrary, no significant correlation was observed between the betweenness centrality based on anatomical connectivity network and the predicted impact on performance $(\mathrm{r}=-0.26, \mathrm{p}=$ 0.14) (Figure-2B). These observations suggest that severity of behavioral impairment induced by a focal lesion can be predicted from the task-evoked connectivity network, but not from the anatomical connectivity network.

\section{Discussion}

In this review, I have contrasted the task-evoked connectivity network with the anatomical connectivity network. In particular, I have demonstrated that, when the former is used, the predicted severity of impairment was proportional to the network hubness ("betweenness centrality") of the computationally lesioned area, while it was not when the latter is used. This result could thus represent a novel approach that enables behavioral impacts of lesions to be predicted using neuroimaging data. Most previous investigations of the hub structure of cortical networks have relied on either structural neuroimaging by diffusion tractography (reflecting anatomical connections) or functional 

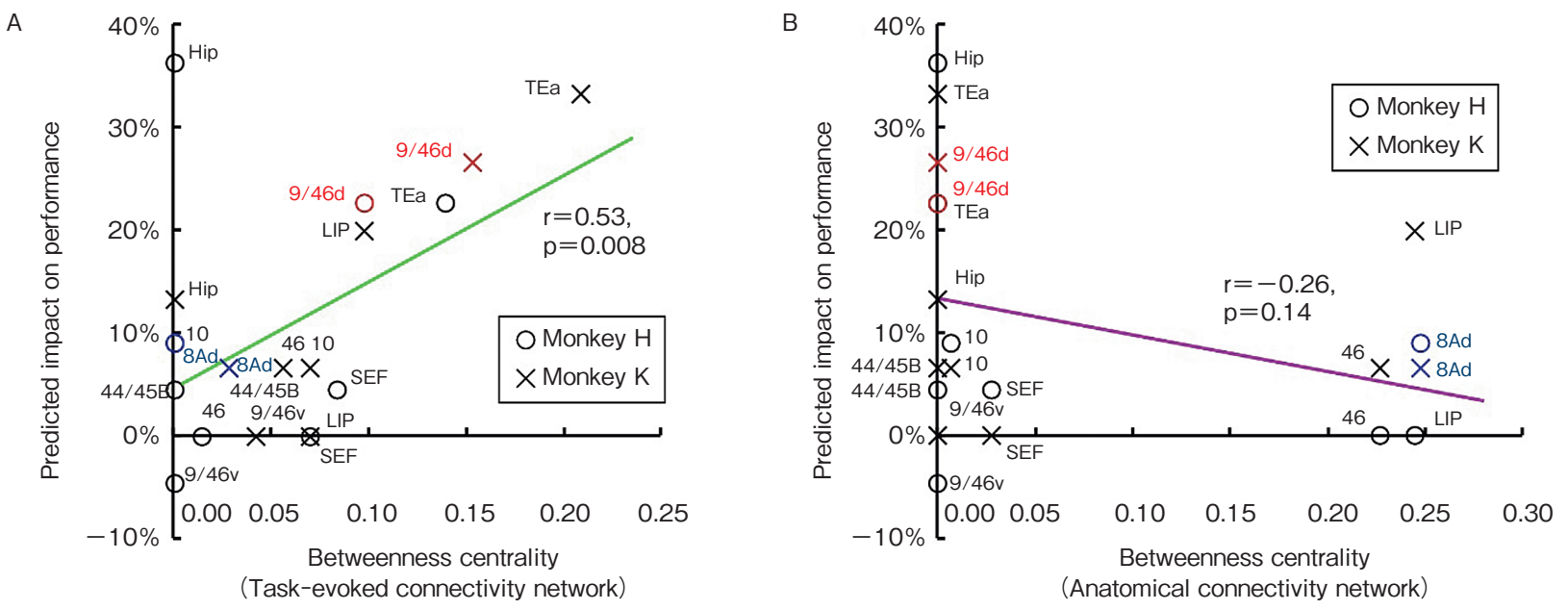

Figure-2 Relationship of betweenness centrality and predicted behavioral impairment after lesioning A. Betweenness centrality was calculated for each area and for each monkey, based on task-evoked connectivity (horizontal axis). Predicted impact on performance (vertical axis) was also calculated. Each o or x represents a value from each area of each monkey. B. Betweenness centrality was calculated based on anatomical connectivity (horizontal axis). Scatter plot is shown as in (A). (Modified from Figure-6A \& B of Osada et al., PLoS Biol, 2015; 13: e1002177 ${ }^{5)}$ )

neuroimaging of spontaneous activity at rest in humans ${ }^{7}$. In addition, the impact of abnormalities in such hubs has been examined in human patients with nonfocal cortical pathologies, such as schizophrenia and Alzheimer's disease ${ }^{11)-13)}$. Thus these previous studies in humans could only investigate brain functions related to general intelligence or general cognitive abilities under the context of static and non-focal brain networks. However, we are usually interested in more specific cognitive abilities and focal brain networks. Indeed, the lesion-effective site is known to vary depending on the cognitive process being engaged ${ }^{2)}{ }^{3)}$ 14) and, as a matter of fact, impairment of a specific function is not always accounted for by structural network hubs. Accordingly, the structural network-based approach is not versatile enough to localize a vulnerable locus for a task-specific cognitive function. Therefore, our novel approach could allow taskevoked fMRI data to be used for the prediction of behavioral impacts of lesions.

I mostly focused in this review on brain functions related to memory tasks, because these are the cutting-edge fields of brain science/neuroscience and we have many previous literatures that attempt to relate brain activity with behavior in this field. When we partake in or watch sports, wider brain areas are incorporated, including subcortical areas such as basal ganglia and cerebellum. Recent advancement of neuroimaging with a high-field MRI scanner at $3 \mathrm{~T}$ or $7 \mathrm{~T}$ has just started to make the analysis of the subcortical areas (such as basal ganglia and cerebellum) in humans possible ${ }^{3)}{ }^{14)}{ }^{15}$. I believe that the approach that I reviewed in this article would be also applicable to the data obtained from those wider brain areas and thus applicable to sportological analyses of behavioral effects that arise when we play or watch sports.

\section{References}

1) Kawamori R: Sportology for the Prevention and Treatment of Diabetes Mellitus. Juntendo Medical Journal, 2010; 56: 239-242.

2) Miyashita Y: Cognitive memory: cellular and network machineries and their top-down control. Science, 2004; 306: 435-440.

3) Hirabayashi T, Miyashita Y: Computational principles of microcircuits for visual object processing in the macaque temporal cortex. Trends Neurosci, 2014; 37: 178187.

4) Miyamoto K, Osada T, Adachi Y, Matsui T, Kimura HM, Miyashita Y: Functional differentiation of memory retrieval network in macaque posterior parietal cortex. Neuron, 2013; 77: 787-799.

5) Osada T, Adachi Y, Miyamoto K, Jimura K, Setsuie R, Miyashita Y: Dynamically allocated hub in task-evoked network predicts the vulnerable prefrontal locus for contextual memory retrieval in macaques. PLoS Biol, 2015; 13: e1002177.

6) Petrides M: Functional specialization within the dorsolateral frontal cortex for serial order memory. Proc R Soc B, 1991; 246: 299-306. 
7) Sporns O: Contributions and challenges for network models in cognitive neuroscience. Nat Neurosci, 2014; 17: $652-660$.

8) Miyashita Y: Neuronal correlate of visual associative long-term memory in the primate temporal cortex. Nature, 1988; 335: 817-820.

9) Miyamoto K, Adachi Y, Osada T, et al: Dissociable memory traces within the macaque medial temporal lobe predict subsequent recognition performance. J Neurosci, 2014; 34: 1988-1997.

10) Stephan KE, Kamper L, Bozkurt A, Burns GA, Young MP, Kötter R: Advanced database methodology for the collation of connectivity data on the macaque brain (CoCoMac). Philos Trans R Soc Lond B Biol Sci, 2001; 356: 1159-1186.

11) Buckner RL, Sepulcre J, Talukdar T, et al: Cortical hubs revealed by intrinsic functional connectivity: Mapping, assessment of stability, and relation to Alzheimer's disease. J Neurosci, 2009; 29: 1860-1873.

12) van den Heuvel MP, Mandl RC, Stam CJ, Kahn RS, Hulshoff Pol HE: Aberrant frontal and temporal complex network structure in schizophrenia: A graph theoretical analysis. J Neurosci, 2010; 30: 15915-15926.

13) Crossley NA, Mechelli A, Scott J, et al: The hubs of the human connectome are generally implicated in the anatomy of brain disorders. Brain, 2014; 137: 2382-2395.

14) Hirabayashi $T$, Takeuchi D, Tamura K, Miyashita Y: Microcircuits for hierarchical elaboration of object coding across primate temporal areas. Science, 2013; 341: 191-195.

15) Watanabe T, Hanajima R, Shirota $Y$, et al: Effects of rTMS of pre-supplementary motor area on frontobasal-ganglia network activity during stop-signal task. J Neurosci, 2015; 35: 4813-4823. 